Journal of Korean Clinical Health Science. http://www.kschs.or.kr

Vol. 2, No 1, March 2014, pp. 1-16

DOI:http://dx.doi.org/10.15205/kschs.2014.2.1.1

\title{
An Analytic Case Study on the Management of an Upper-level General Hospital(2010-2012)
}

\author{
Hyun-Suk Park ${ }^{1 *}$, Jung-Min Lee ${ }^{2}$, Hong-Suck Baek ${ }^{3}$, Jun-Ho Lee ${ }^{3}$, Sang-Sub Park ${ }^{4}$ \\ ${ }^{1 *}$ Department of Health Service Management, Daejeon University \\ ${ }^{2}$ Department of Health Administration, Masan University \\ ${ }^{3}$ Department of Emergency Medical Technology, Daejeon University \\ ${ }^{4}$ Department of Emergency Medical Technology, Chungcheong University \\ (Received January 3, 2014: Revised January 11,2014 :Accepted January 19, 2014)
}

\begin{abstract}
Purpose. For a more efficient hospital management, this study aims to provide basic data so that the hospital management and staff in charge of hospital administration may systematically classify and collect hospital information, by analyzing the ordinary characters of an upper-level general hospital system and its common-type balance sheet, common-type profit and loss statement and financial ratio.

Methods. By using information about an upper-level general hospital in C Province, provided by Alio(www.alio.go.kr), a public organization information provision site, Health Insurance Review \& Assessment Service(www.hira.or.kr) and Ministry of Health and Welfare(www.mw.go.kr), this study analyzed 3 year's data from 2010 to 2012 and provided basic data by analyzing the ordinary characters of an upper-level general hospital system, and its common-type balance sheet, common-type profit and loss statement and financial ratio.
\end{abstract}

Results. After analyzing the ordinary characters, common-type balance sheet, common-type proft and loss statement and financial ration of this general hospital, based on the 2010 to 2012 data, this study came to the following conclusions.

Firstly, out of all the 1,069 hospital staff, there were 272 doctors working for 24 medical departments, out of whom the majority was 33 physicians. Most of the nurses were third-class ones, and about 2,000 outpatients and 600 inpatients on average were treated per day.

Secondly, as a result of analyzing the common-type balance sheet, this study discovered that intangible assets out of fixed assets accounted for $41 \%$, the majority, out of which usable and profitable donation asset buildings were of great importance, and the liquid assets increased more in 2012 than 2011. In the financial structure, the ratio of liquid

\footnotetext{
*Corresponding Author : parkhs@dju.kr
} 
liabilities was over $50 \%$ out of all the liabilities in 2012, and the ratio of purchase payables was high as well. The ratio of fixed liabilities reached up to $40 \%$, out of which the retirement benefit appropriation fund was quite high. The capital was over $80 \%$, but the surplus was in a deficit state. Compared to the capital, the ratio of total liabilities was about $90 \%$, which indicates the financial structure of this general hospital was vulnerable.

Thirdly, as a result of analyzing the common-type profit and loss statement, this study found out that the medical profits from inpatients were higher than profits from outpatients. The material cost was related to the medical quality of this general hospital, and it was as high as $30 \%$ out of the total costs and was about $45 \%$ of the labor cost. This general hospital showed $10 \%$ in the ratio of non-medical profits, and it seemed because of government subsidies. The ratios of medical profits and current net income were gradually changing for the better in 2012, compared to 2011.

Lastly, as a result of analyzing the financial ratio, it was found that the liquidity ratio kept decreasing, from $110.7 \%$ in 2010 and $102.0 \%$ in 2011 to $77.2 \%$ in 2012 . Besides, it was analyzed that the liquidity ratio and the net working capital ratio greatly decreased, while the quick ratio and the liquid ratio kept decreasing.

Conclusions. 1. It is necessary to take the risk management into more consideration, and particularly, it is needed to differentiate and manage the levels of risk in detail. 2. By considering the fact that investments into hospital infrastructures were mostly based on liabilities, it is needed to deal with the scale of losses when evaluating risks. 3. By reflecting the character that investments into hospital infrastructures were based on liabilities, it is necessary to consider the ratio of ordinary profits as well as the ratio of operating profits to sales, and it is also important to consider sales productivity factors, such as the sales amount per a sickbed, by comparing them with other hospitals. As for limitations of this study, there may be some problems in terms of data interpretation because of the lack of information about the number of inpatients and the number of outpatients per year, which are needed for the break-even point analysis. Besides, to suggest a direction for the improvement of hospital management through analyses, non-financial factors should be reflected, such as the trend of economy, medical policies, and politic backgrounds. However, this study only focused on the common-type balance sheet, common-type profit and loss statement and financial ratio, so this study is actually limited to generalizing all the factors by analyzing public data only.

Key words: Common-type balance sheet, Common-type profit and loss statement, Financial ratio analysis, Hospital management analysis 


\section{Introduction}

As a medical organization, a hospital is a management body functioning to provide medical services very different from goods and ordinary services. In Korea, however, the character of medical service, which should be equally assured as public goods and services regardless of patients' conditions, has changed to that of commodities, a target of market trade, since the 1980s, and recently, competition is more emphasized in the medical service industry. As a result, medical service is regarded as commodity, patients as consumers and medical practice as medical industry, and all the hospital should pursue soundness as a management body.

Environmental factors of hospital management are rapidly changing in many ways. Because of more medical organizations and the Korean medical industry open to the world's medical market, competition between medical organizations gets more fierce, and particularly policies controlling medical fees, which are the source of medical profits, are more enforced, so it is inevitable to carry out strategic management fit for each hospital. Accordingly, every hospital should establish effective management strategies, and to make a management strategy fit for each hospital, it is very important to analyze the hospital management, which is one of the basic elements needed to gain a competitive advantage and carry out hospital management successfully. Therefore, planning management strategies for a hospital to accomplish its management objectives should be based on information obtained through hospital management analyses.

In general, hospitals are known as nonprofit or public-interest organizations, and their characters are such various as hospital management environments, medical policies, health insurance institutions, medical consumers and competitive advantage levels against other competing organizations. While external environments are difficult for the hospital management to control, internal environments are possible to control to some degree, so they should change for the better through the rationalization of hospital management for the survival of a hospital ${ }^{1,2}$. As the necessity for the rationalization of hospital management is more emphasized, and all the external environments including the control on medical fees are more deteriorated, every hospital fails to sufficiently produce and analyze hospital information needed to grasp the actual states of hospital management and seek for a better management improvement direction, further leading to unsatisfactory management improvement effects in most cases.

All the reasons for this can be summarized as blow.

Firstly, the management system of a hospital is not rationally designed and operated well enough to properly cope with external environments. Secondly, hospital information needed for an important decision-making process or a rational hospital management is not systematically classified or collected. Thirdly, since the hospital management is mostly composed of doctors, they don't have theoretical knowledge about business management or have poor management techniques. To make things worse, even staff in charge of the administrative management don't have many chances to learn how to manage a 
hospital systematically. Accordingly, this study intends to investigate how the hospital management and staff in charge of the administrative management should classify and collect hospital information systematically and how they should analyze and evaluate the information so that a hospital may be managed more efficiently.

\section{Subjects and Methods}

\subsection{The Necessity of This Study}

First, as an upper-level general hospital located in C Province, this hospital has a management system that is not designed and operated rationally enough to properly cope with external environments. Second, hospital information needed for an important decision-making process or a rational hospital management is not systematically classified, collected or utilized. Third, since the hospital management mostly consists of doctors, they don't have theoretical knowledge about business management or have poor management techniques, and even staff in charge of the administrative management don't have many chances to learn how to manage a hospital systematically. For a more efficient hospital management, therefore, this study aims to help hospital management and staff in charge of the administrative management learn how to classify, collect, analyze and evaluate hospital information systematically.

Based on the information about this upper-level general hospital in C Province, provided by Alio(www.alio.go.kr), a public organization information provision site, Health Insurance Review \& Assessment Service(www.hira.or.kr) and Ministry of
Health and Welfare(www.mw.go.kr), this study analyzed data accumulated from 2010 to 2012 to provide through the ordinary characters of this hospital composition, and the common-type balance sheet, common-type profit and loss statement and financial ration of this hospital.

\subsection{The Purpose of This Study}

The purpose of this study is to provide basic data for the hospital management and staff in charge of administrative management to systematically classify, collect, analyze and evaluate hospital information so that the hospital may be managed more efficiently.

The purpose of this study can be subdivided into the following concrete ones.

1. To investigate the ordinary characters of an upper-level general hospital composition

2. To investigate the common-type balance sheet of an upper-level general hospital

3. To investigate the common-type profit and loss statement of an upper-level general hospital

4. To analyze the financial ratio of an upper-level general hospital

5. To analyze the efficiency through the ordinary characters, common-type balance sheet, common-type profit and loss statement and financial ratio of an upper-level general hospital 


\section{Results}

As shown in Table 1 and 2, the number of medical staffs in this hospital was 1,069, and there were 272 doctors working for 24 different medical departments, out of whom the majority was 33 physicians. Nurses is the third-class, and they attended on about 2,000 outpatients and 600 inpatients on average every day.

As shown in the common-type balance sheet of Table 3, intangible assets out of fixed assets accounted for $41 \%$, the majority, out of which usable and profitable donation asset buildings were of great importance, and the liquid assets increased more in 2012 than 2011. In the financial structure, the ratio of liquid liabilities was over $50 \%$ out of all the liabilities in 2012, and the ratio of purchase payables was high as well. The ratio of fixed liabilities reached up to $40 \%$, out of which the retirement benefit appropriation fund was quite high. The capital was over $80 \%$, but the surplus was in a deficit state. Compared to the capital, the ratio of total liabilities was up to $90 \%$.

As shown in the common-type profit and loss statement of Table 4, the medical profits accounted for $60 \%$, and it was higher than the ratio of profits from outpatients. The material cost was related to the medical quality of this general hospital, and it was as high as $30 \%$ out of the total costs, and about $45 \%$ of the labor cost. This general hospital showed $10 \%$ in the ratio of non-medical profits, and it seemed because of government subsidies. The ratios of medical profits and current net income were gradually changing for the better in 2012, compared to 2011, but still in a deficit state.

As shown in the analysis of the financial ratio of Table 5, the liquidity ratio kept decreasing, from $110.7 \%$ in 2010 and $102.0 \%$ in 2011 to $77.2 \%$ in 2012. Besides, it was analyzed that the liquidity ratio and the net working capital ratio greatly decreased, while the quick ratio and the liquid ratio kept decreasing.

The net working capital ratio kept

Table 1. Actual States of Sickbeds

\begin{tabular}{|c|c|c|c|c|c|c|c|c|c|}
\hline Kinds of & \multicolumn{2}{|c|}{ Wards } & \multicolumn{2}{|c|}{ ICU } & \multirow{2}{*}{$\begin{array}{c}\text { Physical } \\
\text { therapy } \\
\text { room }\end{array}$} & \multirow{2}{*}{$\begin{array}{c}\text { Delivery } \\
\text { room }\end{array}$} & \multirow{2}{*}{$\begin{array}{c}\text { Operating } \\
\text { room }\end{array}$} & \multirow{2}{*}{$\begin{array}{c}\text { Emerge } \\
\text { ncy } \\
\text { room }\end{array}$} & \multirow[b]{2}{*}{ Total } \\
\hline \multirow[t]{2}{*}{$\begin{array}{l}\text { Section/ } \\
\text { No. of } \\
\text { Sickbeds }\end{array}$} & $\begin{array}{l}\text { Adva } \\
\text { nced }\end{array}$ & $\begin{array}{l}\text { Ordin } \\
\text { ary }\end{array}$ & $\begin{array}{l}\text { Adult } \\
\text { Infant/ } \\
\text { Teens }\end{array}$ & $\begin{array}{l}\text { New } \\
\text { born }\end{array}$ & & & & & \\
\hline & 129 & 455 & 29 & 25 & 22 & 2 & 10 & 30 & 702 \\
\hline
\end{tabular}

* As one of the characters, this hospital has 702 sickbeds (including special parts).

Table 2. Actual States of the Medical Staff

\begin{tabular}{|c|c|c|c|c|c|c|c|c|c|}
\hline Job & Executive & \multicolumn{5}{|c|}{ Doctor } & $\begin{array}{c}\text { Nurses and } \\
\text { ordinary }\end{array}$ & $\begin{array}{c}\text { Adminis } \\
\text { tration }\end{array}$ & Total \\
\hline Section & \multirow[t]{2}{*}{1} & $\begin{array}{c}\text { Professors } \\
\text { with } \\
\text { additional } \\
\text { posts } \\
\end{array}$ & $\begin{array}{c}\text { Clinical } \\
\text { professors }\end{array}$ & $\begin{array}{c}\text { Full-time } \\
\text { doctors }\end{array}$ & Residents & $\begin{array}{c}\text { Contracted } \\
\text { doctors }\end{array}$ & \multirow[t]{2}{*}{600} & \multirow[t]{2}{*}{186} & \multirow[t]{2}{*}{1069} \\
\hline Number & & 98 & 22 & 10 & 151 & 1 & & & \\
\hline
\end{tabular}


Table 3. A Common-type Balance Sheet

(Unit: Won)

\begin{tabular}{|c|c|c|c|}
\hline Account Title & 2012 & 2011 & 2010 \\
\hline I. Medical Profits & $100.00 \%$ & $100.00 \%$ & $100.00 \%$ \\
\hline 1. Medical Profits & $100.00 \%$ & $100.00 \%$ & $100.00 \%$ \\
\hline Profits from Inpatients & $58.25 \%$ & $58.96 \%$ & $59.28 \%$ \\
\hline Profits from Outpatients & $39.75 \%$ & $39.33 \%$ & $38.73 \%$ \\
\hline Other Medical Profits & $2.00 \%$ & $1.71 \%$ & $1.99 \%$ \\
\hline П. $\quad$ Medical Costs & $103.96 \%$ & $109.04 \%$ & $103.45 \%$ \\
\hline 1. Material Cost & $32.72 \%$ & $32.53 \%$ & $33.34 \%$ \\
\hline Medicine Cost & $17.23 \%$ & $17.26 \%$ & $18.95 \%$ \\
\hline Treatment Material Cost & $14.49 \%$ & $14.14 \%$ & $13.32 \%$ \\
\hline Medical Consumable Cost & $0.22 \%$ & $0.34 \%$ & $0.30 \%$ \\
\hline Meal Material Cost & $0.78 \%$ & $0.78 \%$ & $0.76 \%$ \\
\hline 2. Labor Costs & $44.56 \%$ & $48.42 \%$ & $44.38 \%$ \\
\hline Wages & $14.51 \%$ & $15.48 \%$ & $14.89 \%$ \\
\hline Allowances & $21.99 \%$ & $23.69 \%$ & $22.42 \%$ \\
\hline Wages for Temporary Employees & $2.93 \%$ & $2.14 \%$ & $1.33 \%$ \\
\hline Retirement Allowances & $5.12 \%$ & $7.11 \%$ & $5.74 \%$ \\
\hline 3. Management Costs & $26.68 \%$ & $28.09 \%$ & $25.73 \%$ \\
\hline Welfare Benefits & $5.05 \%$ & $4.79 \%$ & $4.16 \%$ \\
\hline Traveling and Transportation Cost & $0.01 \%$ & $0.01 \%$ & $0.02 \%$ \\
\hline Communication Cost & $0.11 \%$ & $0.12 \%$ & $0.12 \%$ \\
\hline Power and Water Cost & $1.32 \%$ & $1.39 \%$ & $1.32 \%$ \\
\hline Fuel Cost & $0.93 \%$ & $0.98 \%$ & $1.00 \%$ \\
\hline Tax and Utility Bills & $0.07 \%$ & $0.06 \%$ & $0.07 \%$ \\
\hline Consumable Cost & $0.59 \%$ & $0.75 \%$ & $0.77 \%$ \\
\hline Clothing \& Bedding Cost & $0.53 \%$ & $0.51 \%$ & $0.46 \%$ \\
\hline Book and Printing Cost & $0.12 \%$ & $0.15 \%$ & $0.13 \%$ \\
\hline Depreciation Cost & $5.35 \%$ & $6.27 \%$ & $5.55 \%$ \\
\hline Ontangible Asset Depreciation Cost & $0.91 \%$ & $0.95 \%$ & $0.85 \%$ \\
\hline Facility Maintenance Cost & $0.95 \%$ & $1.72 \%$ & $1.47 \%$ \\
\hline Vehicle Operation Cost & $0.02 \%$ & $0.02 \%$ & $0.02 \%$ \\
\hline Insurance Premium & $0.02 \%$ & $0.02 \%$ & $0.02 \%$ \\
\hline Rent \& Commission & $0.70 \%$ & $0.81 \%$ & $0.67 \%$ \\
\hline Business-operating Cost & $0.26 \%$ & $0.29 \%$ & $0.31 \%$ \\
\hline Public Relation Cost & $0.10 \%$ & $0.12 \%$ & $0.08 \%$ \\
\hline Education \& Training Cost & $0.08 \%$ & $0.08 \%$ & $0.11 \%$ \\
\hline Investigation \& Analysis Cost & $0.02 \%$ & $0.02 \%$ & $0.02 \%$ \\
\hline Rewards & $0.03 \%$ & $0.03 \%$ & $0.01 \%$ \\
\hline Medical Community Project Cost & $0.03 \%$ & $0.07 \%$ & $0.04 \%$ \\
\hline Bad Debt Cost & $0.05 \%$ & $0.10 \%$ & $0.08 \%$ \\
\hline Premium Treatment Allowance & $3.44 \%$ & $3.41 \%$ & $3.28 \%$ \\
\hline Premium Treatment Research Cost & $0.51 \%$ & $0.57 \%$ & $0.58 \%$ \\
\hline Premium Treatment-related Expense & $0.74 \%$ & $0.80 \%$ & $1.07 \%$ \\
\hline Compensation for Malpractices & $0.09 \%$ & $0.10 \%$ & $0.11 \%$ \\
\hline Clinical Research Cost & $0.28 \%$ & $0.30 \%$ & $0.31 \%$ \\
\hline Research Book Cost & $0.04 \%$ & $0.04 \%$ & $0.02 \%$ \\
\hline Medical Education \& Training Cost & $0.15 \%$ & $0.12 \%$ & $0.12 \%$ \\
\hline Educational Practice Cost & $0.02 \%$ & $0.02 \%$ & $0.01 \%$ \\
\hline Research Theses Cost & $0.01 \%$ & $0.01 \%$ & $0.00 \%$ \\
\hline Compensations & $0.71 \%$ & $0.00 \%$ & $0.00 \%$ \\
\hline
\end{tabular}




\begin{tabular}{|c|c|c|c|}
\hline Outsourcing Service Cost & $3.19 \%$ & $3.20 \%$ & $2.86 \%$ \\
\hline Other Expenses & $0.27 \%$ & $0.27 \%$ & $0.10 \%$ \\
\hline Medical Profits & $-3.96 \%$ & $-9.04 \%$ & $-3.45 \%$ \\
\hline IV. Non-medical Profits & $7.15 \%$ & $6.49 \%$ & $5.91 \%$ \\
\hline 1. Incidental Medical Profits & $3.13 \%$ & $3.22 \%$ & $2.52 \%$ \\
\hline 2. Profits from Interest & $0.16 \%$ & $0.09 \%$ & $0.45 \%$ \\
\hline 3. Profits from Tangible Asset Disposition & $0.00 \%$ & $0.00 \%$ & $0.00 \%$ \\
\hline 4. Profits from the Modification of Power Bill Errors & $0.27 \%$ & $0.01 \%$ & $0.00 \%$ \\
\hline 5. Profits from Outsourcing Research & $1.67 \%$ & $1.20 \%$ & $0.85 \%$ \\
\hline 6. Donation Profits & $0.09 \%$ & $0.08 \%$ & $0.04 \%$ \\
\hline 7. Rent & $0.04 \%$ & $0.04 \%$ & $0.04 \%$ \\
\hline 8. Other Project Profits & $1.46 \%$ & $1.47 \%$ & $1.63 \%$ \\
\hline 9. Miscellaneous Profits & $0.33 \%$ & $0.38 \%$ & $0.37 \%$ \\
\hline V. $\quad$ Non-medical Cost & $6.38 \%$ & $5.16 \%$ & $4.74 \%$ \\
\hline 1. Incidental Medical Cost & $1.22 \%$ & $1.26 \%$ & $0.58 \%$ \\
\hline 2. Cost for Interest & $0.41 \%$ & $0.16 \%$ & $0.24 \%$ \\
\hline 3. Loss from the Modification of Power Bill Errors & $0.69 \%$ & $0.05 \%$ & $0.00 \%$ \\
\hline 4. Loss from Inventory Asset Disposition & $0.00 \%$ & $0.00 \%$ & $0.00 \%$ \\
\hline 5. Loss from Repayment of Foreign Borrowing & $0.00 \%$ & $0.00 \%$ & $0.00 \%$ \\
\hline 6. Donation & $0.05 \%$ & $0.04 \%$ & $0.05 \%$ \\
\hline 7. Loss from Tangible Asset Disposition & $0.00 \%$ & $0.00 \%$ & $0.01 \%$ \\
\hline 8. Outsourcing Research Cost & $1.58 \%$ & $1.11 \%$ & $0.81 \%$ \\
\hline 9. Costs for Other Projects & $2.42 \%$ & $2.52 \%$ & $1.98 \%$ \\
\hline 10. Essential Business Reserve & $0.00 \%$ & $0.00 \%$ & $0.89 \%$ \\
\hline 11. Miscellaneous Loss & $0.01 \%$ & $0.01 \%$ & $0.19 \%$ \\
\hline VI. Ordinary Profits & $-3.19 \%$ & $-7.71 \%$ & $-2.29 \%$ \\
\hline VII. Special Profits & $0.01 \%$ & $0.09 \%$ & $0.16 \%$ \\
\hline 1. Profits from Assets Contributed & $0.01 \%$ & $0.09 \%$ & $0.16 \%$ \\
\hline VIII. Special Losses & $0.00 \%$ & $0.00 \%$ & $0.00 \%$ \\
\hline 1. Other Special Losses & $0.00 \%$ & $0.00 \%$ & $0.00 \%$ \\
\hline IX. Corporate Tax and So on & $0.00 \%$ & $0.00 \%$ & $0.00 \%$ \\
\hline 1. Cost for Corporate Tax & $0.00 \%$ & $0.00 \%$ & $0.00 \%$ \\
\hline X. $\quad$ Current Net Profits & $-3.18 \%$ & $-7.63 \%$ & $-2.13 \%$ \\
\hline
\end{tabular}

Table 4. A Common-type Profit and Loss Statement

\begin{tabular}{c|r|r|r}
\hline \multicolumn{1}{c|}{ Account Title } & \multicolumn{1}{c}{ (Unit: \%) } \\
\hline Assets & & 2012 & 2010 \\
I. Liquid Assets & $39.47 \%$ & $33.58 \%$ & $38.26 \%$ \\
1. Quick Assets & $37.74 \%$ & $32.48 \%$ & $37.67 \%$ \\
Cash \& Cash Equivalents & $7.22 \%$ & $6.54 \%$ & $7.14 \%$ \\
Short-term Financial Instruments & $7.65 \%$ & $4.03 \%$ & $16.47 \%$ \\
Negotiable Securities & $0.00 \%$ & $0.00 \%$ & $0.01 \%$ \\
(Allowance for Bad Debts) & $18.65 \%$ & $16.02 \%$ & $13.41 \%$ \\
Other Receivables & $-0.79 \%$ & $-0.77 \%$ & $-0.65 \%$ \\
(Allowance for Bad Debts) & $0.82 \%$ & $0.64 \%$ & $0.63 \%$ \\
Receivable Profits & $0.00 \%$ & $0.00 \%$ & $0.00 \%$ \\
Advanced Payment & $0.11 \%$ & $0.05 \%$ & $0.14 \%$ \\
2. Inventory Assets & $4.09 \%$ & $5.98 \%$ & $0.52 \%$ \\
Medicine for Injection & $1.73 \%$ & $1.10 \%$ & $0.59 \%$ \\
Drugs & $0.91 \%$ & $0.47 \%$ & $0.14 \%$
\end{tabular}




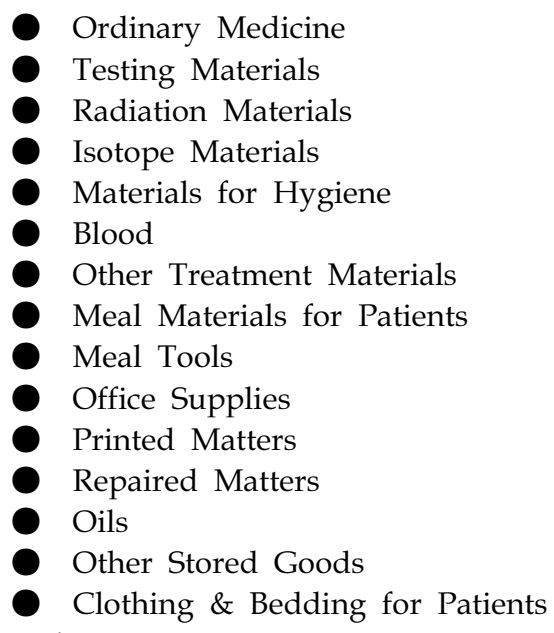

П. Fixed Assets

1. Investment Assets

- Security Deposits

2. Tangible Assets

- Structures

(Cumulative Amount of Depreciation)

- Machinery Devices

(Cumulative Amount of Depreciation)

- Vehicle Delivery Equipment

(Cumulative Amount of Depreciation)

- Medical Devices

(Cumulative Amount of Depreciation)

- Tools and Instruments

(Cumulative Amount of Depreciation)

- Furniture for Medical Use

(Cumulative Amount of Depreciation)

- Ordinary Furniture

(Cumulative Amount of Depreciation)

- Financial Lease Assets

(Cumulative Amount of Depreciation)

- Assets under Construction

3. Intangible Assets

- Usable \& Profitable Donation Asset Buildings

Other Intangible Assets

A Total of Assets

\section{Liabilities}

1. Liquid Liabilities

- Purchase Liabilities

- Account Payables

- Short-term Debts

- Liquid Long-term Liabilities

- Advanced Payment

- Liquid Deposit Received

- Unearned Profits

- Non-liquid Deposit Received

П. Fixed Liabilities

- Long-term Debts

\begin{tabular}{|c|c|c|}
\hline $0.41 \%$ & $0.24 \%$ & $0.10 \%$ \\
\hline $0.16 \%$ & $0.13 \%$ & $0.09 \%$ \\
\hline $0.02 \%$ & $0.02 \%$ & $0.03 \%$ \\
\hline $0.02 \%$ & $0.02 \%$ & $0.01 \%$ \\
\hline $0.02 \%$ & $0.03 \%$ & $0.02 \%$ \\
\hline $0.01 \%$ & $0.01 \%$ & $0.01 \%$ \\
\hline $0.00 \%$ & $0.00 \%$ & $0.00 \%$ \\
\hline $0.00 \%$ & $0.00 \%$ & $0.00 \%$ \\
\hline $0.01 \%$ & $0.01 \%$ & $0.01 \%$ \\
\hline $0.00 \%$ & $0.01 \%$ & $0.02 \%$ \\
\hline $0.00 \%$ & $0.00 \%$ & $0.01 \%$ \\
\hline $0.09 \%$ & $0.08 \%$ & $0.09 \%$ \\
\hline $0.00 \%$ & $0.00 \%$ & $0.00 \%$ \\
\hline $0.00 \%$ & $0.01 \%$ & $0.01 \%$ \\
\hline $0.00 \%$ & $0.00 \%$ & $0.00 \%$ \\
\hline $62.26 \%$ & $66.42 \%$ & $61.74 \%$ \\
\hline $0.01 \%$ & $0.01 \%$ & $0.01 \%$ \\
\hline $0.01 \%$ & $0.01 \%$ & $0.01 \%$ \\
\hline $20.57 \%$ & $22.89 \%$ & $27.86 \%$ \\
\hline $1.45 \%$ & $1.47 \%$ & $1.40 \%$ \\
\hline$-0.46 \%$ & $-0.39 \%$ & $-0.31 \%$ \\
\hline $6.56 \%$ & $6.31 \%$ & $5.33 \%$ \\
\hline$-5.35 \%$ & $-4.66 \%$ & $-3.71 \%$ \\
\hline $0.16 \%$ & $0.16 \%$ & $0.15 \%$ \\
\hline$-0.16 \%$ & $-0.14 \%$ & $-0.12 \%$ \\
\hline $46.99 \%$ & $47.38 \%$ & $40.46 \%$ \\
\hline$-35.60 \%$ & $-31.07 \%$ & $-25.02 \%$ \\
\hline $0.02 \%$ & $0.02 \%$ & $0.02 \%$ \\
\hline$-0.02 \%$ & $-0.02 \%$ & $-0.01 \%$ \\
\hline $0.74 \%$ & $0.79 \%$ & $0.81 \%$ \\
\hline$-0.71 \%$ & $-0.72 \%$ & $-0.70 \%$ \\
\hline $3.47 \%$ & $3.46 \%$ & $3.09 \%$ \\
\hline$-2.64 \%$ & $-2.41 \%$ & $-1.97 \%$ \\
\hline $5.98 \%$ & $9.22 \%$ & $7.20 \%$ \\
\hline$-4.97 \%$ & $-7.86 \%$ & $-7.17 \%$ \\
\hline $5.12 \%$ & $1.36 \%$ & $8.40 \%$ \\
\hline $41.68 \%$ & $43.52 \%$ & $33.87 \%$ \\
\hline $41.12 \%$ & $42.94 \%$ & $33.33 \%$ \\
\hline $0.57 \%$ & $0.57 \%$ & $0.55 \%$ \\
\hline $100.00 \%$ & $100.00 \%$ & $100.00 \%$ \\
\hline $50.25 \%$ & $32.92 \%$ & $34.53 \%$ \\
\hline $24.39 \%$ & $22.70 \%$ & $22.64 \%$ \\
\hline $7.96 \%$ & $5.05 \%$ & $6.59 \%$ \\
\hline $12.98 \%$ & $0.00 \%$ & $0.00 \%$ \\
\hline $0.55 \%$ & $0.78 \%$ & $1.98 \%$ \\
\hline $0.94 \%$ & $1.02 \%$ & $0.52 \%$ \\
\hline $0.49 \%$ & $0.59 \%$ & $0.43 \%$ \\
\hline $2.19 \%$ & $2.15 \%$ & $1.87 \%$ \\
\hline $0.75 \%$ & $0.63 \%$ & $0.51 \%$ \\
\hline $39.46 \%$ & $50.69 \%$ & $42.16 \%$ \\
\hline $1.81 \%$ & $2.11 \%$ & $2.45 \%$ \\
\hline
\end{tabular}


- Financial Lease Account Payables

- Retirement Allowances

(Retirement Allowance Converted to the National Pension)

(Deposits for Retirement Insurance)

Other Long-term Allowances

- Reserve Fund for Essential Business

- Reserve Fund for Medical Development A Grand Total of Liabilities

Capital

I . Capital Funds

(1) Capital Funds

- Corporate Endowment

Other Endowments

Contribution

Subsidies

П. Surplus

(1) Capital Surplus

- Other Surpluses

(2) Earned Surplus

- Surplus to be Carried Forward

(Net Loss during the Current Term: $\triangle 4,082,672,552$ won)

A Total of Capital

\begin{tabular}{r|r|r}
$0.74 \%$ & $1.10 \%$ & $0.00 \%$ \\
$36.13 \%$ & $47.43 \%$ & $39.56 \%$ \\
$-0.23 \%$ & $-0.37 \%$ & $-0.38 \%$ \\
$-0.63 \%$ & $-0.63 \%$ & $-0.59 \%$ \\
$0.63 \%$ & $0.01 \%$ & $0.11 \%$ \\
$0.00 \%$ & $0.00 \%$ & $1.00 \%$ \\
$1.02 \%$ & $1.05 \%$ & $0.00 \%$ \\
$89.71 \%$ & $83.61 \%$ & $76.69 \%$ \\
$0.00 \%$ & $0.00 \%$ & $0.00 \%$ \\
$85.36 \%$ & $87.63 \%$ & $82.34 \%$ \\
$85.36 \%$ & $87.63 \%$ & $82.34 \%$ \\
$19.88 \%$ & $20.52 \%$ & $19.55 \%$ \\
$65.48 \%$ & $67.10 \%$ & $62.78 \%$ \\
$29.99 \%$ & $30.95 \%$ & $29.49 \%$ \\
$35.49 \%$ & $36.16 \%$ & $33.30 \%$ \\
$-75.07 \%$ & $-71.24 \%$ & $-59.03 \%$ \\
$0.00 \%$ & $0.00 \%$ & $0.00 \%$ \\
$0.00 \%$ & $0.00 \%$ & $0.00 \%$ \\
$-75.07 \%$ & $-71.24 \%$ & $-59.03 \%$ \\
$-75.07 \%$ & $-71.24 \%$ & $-59.03 \%$ \\
$0.00 \%$ & $0.00 \%$ & $0.00 \%$ \\
$10.29 \%$ & $16.39 \%$ & $23.31 \%$ \\
\hline $100.00 \%$ & $100.00 \%$ & $100.00 \%$ \\
\hline
\end{tabular}

A Grand Total of Liabilities and Capital

Source: (www.alio.go.kr),(www.hira.or.kr),(www.mw.go.kr).

greatly decreasing from $3.7 \%$ and $0.7 \%$ to $-11.4 \%$ for the three years. The medical cost defensive period decreased from 125.2 days in 2010 to 94.8 days in 2011, but greatly increased to 348.8 days in 2012 .

The liability ratio of this hospital kept increasing from $329.1 \%$ and $510.1 \%$ to $872.1 \%$ for the three years. The equity capital ratio, however, kept decreasing

Table 5. An Analysis of the Financial Ratio from $23.3 \%$ in 2010 and $16.4 \%$ in 2011 to $10.3 \%$ in 2012. The interest earned ratio was 10.7 times in 2010, and greatly increased to 48 times in 2011, and the fixed assets to long-term capital ratio was less than $100 \%$, while being invested into fixed assets within the range of equity capital and fixed liabilities.

\begin{tabular}{|c|c|c|c|}
\hline & & & (Unit: \%) \\
\hline Account Title & 2012 & 2011 & 2010 \\
\hline \multicolumn{4}{|l|}{ 1. Liquidity Ratio } \\
\hline Liquid Ratio (\%) & 77.2 & 102.0 & 110.7 \\
\hline Qucik Ratio (\%) & 73.8 & 98.7 & 109.1 \\
\hline Net Working Capital Composition Ratio (\%) & -11.4 & 0.7 & 3.7 \\
\hline Medical Cost Defensive Period (Days) & 384.8 & 94.8 & 125.2 \\
\hline \multicolumn{4}{|l|}{ 2. Leverage Ratio } \\
\hline Debt Ratio (\%) & 872.1 & 510.1 & 329.1 \\
\hline Equity Capital Ratio (\%) & 10.3 & 16.4 & 23.3 \\
\hline
\end{tabular}




\begin{tabular}{|c|c|c|c|}
\hline Interest Earned Ratio (Times) & 8.7 & 48 & 10.7 \\
\hline Fixed Ratio $(\%)$ & 594.9 & 405.2 & 264.9 \\
\hline Fixed Assets to Long-term Capital Ratio (\%) & 123.0 & 99.0 & 94.3 \\
\hline \multicolumn{4}{|l|}{ 3. Profitability Ratio } \\
\hline Medical Return on Medical Profits (\%) & -3.95916 & -9.03771 & -3.45259 \\
\hline Net Return on Medical Profits (\%) & -3.18077 & -7.62537 & -2.13191 \\
\hline Medical Return on Total Assets (\%) & -5.26482 & -10.99734 & -3.87310 \\
\hline Net Return on Total Assets (\%) & -4.22972 & -9.27877 & -2.39156 \\
\hline Net Return on Equity Capital (\%) & -40.49656 & -57.26736 & -11.00835 \\
\hline \multicolumn{4}{|l|}{ 4. Growth Ratio } \\
\hline Increase Ratio of Profits from Inpatients (\%) & 9.534820462 & 2.79954209 & 2.140715593 \\
\hline Increase Rate of Profits from Outpatients (\%) & 12.05081152 & 4.941353678 & 12.3269263 \\
\hline Increase Ratio of Total Assets (\%) & 1.45248138 & -4.725095143 & 7.330553799 \\
\hline Increase Rate of Net Profits (\%) & -53.75292197 & 269.6475238 & 68.59085827 \\
\hline \multicolumn{4}{|l|}{ 5. Activity Ratio } \\
\hline Total Asset Turnover Ratio (\%) & 1.121792964 & 1.2168 & 1.3071 \\
\hline Tangible Asset Turnover Ratio (\%) & 4.026778496 & 5.3152 & 6.4649 \\
\hline Medical Account Receivable Turnover Ratio (\%) & 8.366373929 & 7.595 & 7.132 \\
\hline Purchase Liability Turnover Ratio (\%) & 4.95549016 & 7.2117 & 5.358 \\
\hline Inventory Asset Turnover Ratio (\%) & 191.212421 & 110.726 & 76.783 \\
\hline \multicolumn{4}{|l|}{ 6. Productivity Ratio } \\
\hline Value Added (1,000 won) & 61274260977 & 55480698190 & 53964293067 \\
\hline Value-Added Ratio (\%) & 47.67102794 & 47.92285165 & 48.17285552 \\
\hline Total Capital Investment Efficiency (\%) & 606.610566 & 355.7847685 & 231.8663727 \\
\hline Added Value per Employee (1,000 won) & 57319233.84 & 51899624.13 & 50481097.35 \\
\hline
\end{tabular}

Implications

1. It is necessary to take the risk management into careful consideration and differentiate the levels of risk management fit for local hospitals

2. It is needed to consider the scale of losses prudently while reflecting the fact that infrastructure investments are based on liabilities when evaluating risks

3. It is needed to consider the ratio of ordinary profits as well as the ratio of operating profits to sales by reflecting the fact that hospital infrastructure investments are based on liabilities. Besides, it is also important to consider sales productivity factors, such as the sales amount per a sickbed, while comparing them with other hospitals.

As for limitations of this study, there may be some problems in terms of data interpretation because of the lack of information about the number of inpatients and outpatients per year, which is needed for the break-even point analysis. Besides, to suggest a direction for the improvement of hospital management through analyses, non-financial factors, should be reflected, such as the trend of economy, medical policies and politic backgrounds. However, this study focused only on the commontype balance sheet, common-type profit and loss statement and financial ratio of this general hospital. Actually, this study is limited to generalizing all the data with information open only. Therefore, further 


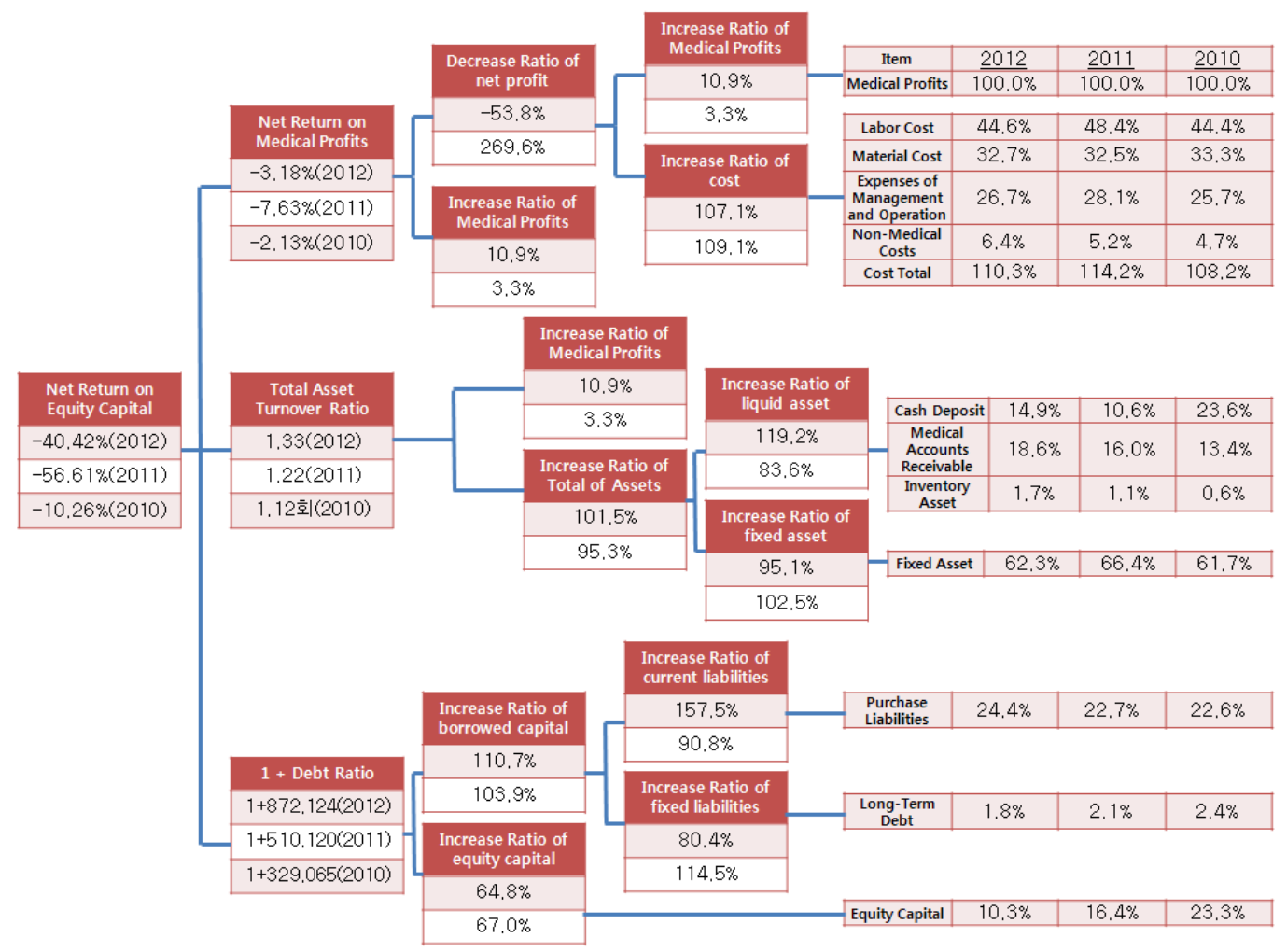

Figure 1. A Systematic Diagram for ROI Analysis

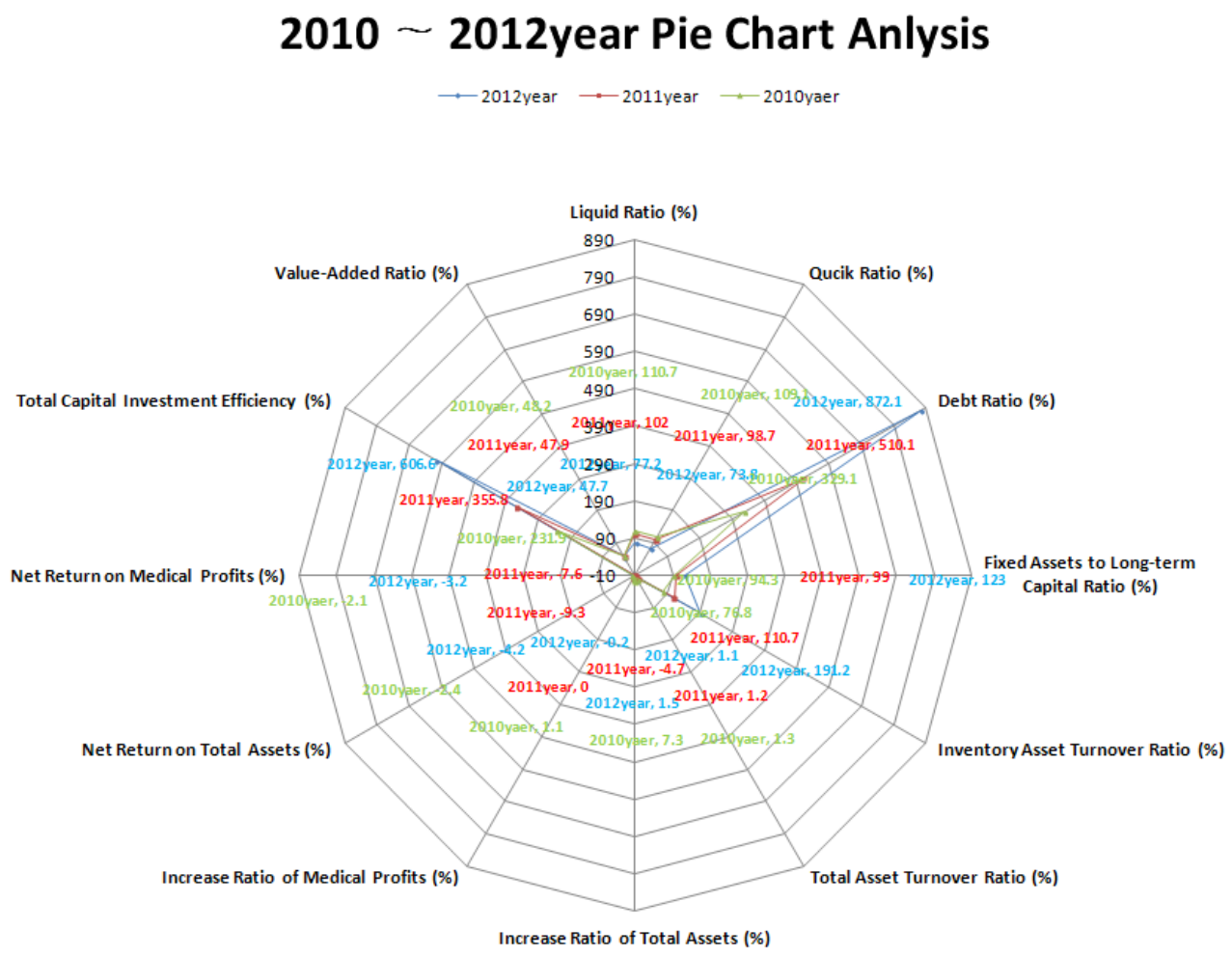

Figure 2. A Circular Diagram for the Financial Ratio from 2010 to 2012 
studies should be carried out with non-financial factors.

As shown in the circular diagram for the financial ratio in 2010, it was found that the increase ratio of total assets and the net return on medical profits, etc. were relatively low, while the value added ratio, the total asset investment efficiency and the increase ratio of medical profits, etc. were relatively stable.

In the circular diagram for the financial ratio in 2011, the total asset investment efficiency and the increase ratio of medical profits, etc. greatly decreased, while the ratio of liabilities increased, compared to 2010. It seemed mainly because of the introduction of MRI devices, and this introduction of new devices brought about effect of raising the increase ratio of total assets. At the same time, it seemed to contribute to increasing the net return on medical profits by a small margin.

In the circular diagram for the financial ratio in 2012, the most remarkable change is the reduction of the net return on medical profits, compared to 2011. It seemed because of a rapid increase in the welfare \& benefit costs for employees and research costs, and the reason why the total asset investment efficiency became lower seemed to be because of problems related to device application capabilities after the introduction of new devices.

\section{Review}

In general, an analysis of hospital management is carried out by using the balance sheet, profit and loss statement, patient treatment performance, medical profits, human resources for each job field, facility use performance and current circumstances and so $\mathrm{on}^{3-7)}$. As for the significance of a hospital management analysis, the management capability and financial states of a hospital can be analyzed on the basis of extensive financial data, including the past, present and future plans, and management statistic figures about patient treatment performance, etc, and it is also a general term for all the analysis methods used to provide useful management information to the persons concerned, including a CEO, by verifying all the reasons ${ }^{3-7)}$. When reviewing previous researches to analyze the relation between the publicness and profitability of national university hospitals, this study found out that there are some differences in results, depending on the kind of measurement index, and since they are not standardized, it was difficult to explain the analysis results ${ }^{8)}$.

Firstly, as for the ordinary characters of this upper-level general hospital, there were a total of 1,069 employees working for this hospital, and 272 doctors working for 24 medical departments, out of whom the majority was 33 physicians. Most of the nurses were third-class ones, and about 2,000 outpatients and 600 inpatients on average were treated per day. The reason why there were large differences between hospitals in reality when analyzing whether the number of employees was excessive is because there was a difference in the number of nurses, depending on the nurses' class ${ }^{9)}$.

Secondly, as a result of analyzing the common-type balance sheet, this study found out that intangible assets out of fixed assets accounted for $41 \%$, the majority, out of which usable and 
profitable donation asset buildings were of great importance, and the liquid assets increased more in 2012 than 2011. It seemed to be because of an increase in the ratio of quick assets, especially, cash \& cash equivalents and short-term financial instruments. In the financial structure, the ratio of liquid liabilities was over $50 \%$ out of all the liabilities in 2012, and especially, the ratio of purchase payables was really high. The ratio of fixed liabilities reached up to $40 \%$, out of which the retirement benefit appropriation fund was quite high. The capital was over $80 \%$, but the surplus was in a deficit state. Compared to the capital, the ratio of total liabilities was about $90 \%$, which indicates the financial structure of this general hospital was vulnerable. Besides, as a result of carrying out a complete enumeration survey for about 2,800 hospital-ranked medical organizations all over the country (ordinary hospitals, mental hospitals, infectionspecialized hospitals, oriental medicine clinics, and dental clinics, etc.), this study found out that the financial structure of hospitals was somewhat deteriorated in 2012, compared to 2011, and the income and expenditure of the medical industry is still in a vulnerable state, compared to other industries (like the manufacturing industry), while being more deteriorated than 2010. Besides, the medical profits were found to increase a little, compared to the previous year, but it was all because of an increase in labor cost, etc. In short, the financial structure of hospitals, such as equity capital, is still in a vulnerable state, compared to other industries, but medical prime costs tend to increase, such as labor $\operatorname{cost}^{6}$. As for the actual states of hospital management in 2011, the total profits decreased, compared to the previous year, despite an increase in the medical fee $(2.05 \%$ on Jan. 1,2010 to $1.64 \%$ on Jan. 1, 2011) and a partial increase in the medical profits ${ }^{4.6)}$. In Korea, hospitals used to depend on medical profits through patient treatment before. Due to the governmental control on the medical fee, various regulations on the medical service industry and more fierce competition accompanied by an increase in the number of hospitals, however, they have fewer medical profits, leading to more difficulty in hospital management ${ }^{10)}$.

Thirdly, as a result of analyzing the common-type profit and loss statement, this study found out that medical profits from inpatients were higher than profits from outpatients. The material cost was related to the medical quality of this general hospital, and it was as high as $30 \%$ out of the total costs, which indicates that this hospital provides patients with high-quality medical service to some degree. However, the material cost increased up to $45 \%$ of the labor cost, leading to a deficit state. This general hospital showed $10 \%$ in the ratio of non-medical profits, and it seemed because of government subsidies. The ratios of medical profits and current net profits were gradually changing for the better in 2012, compared to 2011. However, as they are still in a difficult deficit state, it is urgent to improve this condition. Yang Jong-hyeon et, al. carried out a research on the main hospital management performances from 2008 to 2010. Through the research results, how the financial ratio of hospitals changed for the three years, 
particularly in the medical profits, medical costs, non-medical profits, non-medical costs, net profits during the term, wages for outpatient treatments, medical return on medical profits, net return on medical profits and ordinary return on medical profits. Especially, the current net profits of this hospital seem quite similar to those of other national university hospitals ${ }^{10,15)}$.

Fourthly, as a result of analyzing the financial ratio, this study discovered that the liquidity ratio kept decreasing, from $110.7 \%$ in 2010 and $102.0 \%$ in 2011 to $77.2 \%$ in 2012. It was because the shot-term debt sharply increased, further increasing the liquid liabilities. Besides, it was analyzed that the liquidity ratio and the net working capital ratio greatly decreased, because the shot-term debt sharply increased, further increasing the liquid liabilities. According to Lee Eun-hyeong' case study, only C Hospital showed favorable aspects by exceeding the ordinary level of soundness, $150 \%$, while the other 3 hospitals failed to reach 100\%, showing a very low liquidity. Especially, B Hospital didn't even reach up to $50 \%$, being judged to suffer from a sever monetary stringency ${ }^{9-10,15-16)}$.

As the quick ratio and liquid ratio kept decreasing, both of which were rather favorable, compared to the industrial average ratio, it was analyzed that this hospital is capable to redeem short-term debts. Even in Lee Eun-hyeong's case study on the quick ratio of hospitals, C Hospital showed favorable aspects, but the remaining hospitals were found not to reach $76 \%$, which is the average ratio of hospital in urban areas. Especially, the quick ratio of $\mathrm{B}$ Hospital was as low as
$30.1 \%$, being analyzed to have a poor capability to redeem short-term debts ${ }^{9-16)}$.

The net working capital ratio of this subject hospital kept greatly decreasing from $3.7 \%$ and $0.7 \%$ to $-11.4 \%$ for the three years. The reason for this decrease for the three years is because the liquid assets greatly decreased as the medical accounts receivable decreased, and shortterm financial instruments were purchased. The medical cost defensive period decreased from 125.2 days in 2010 to 94.8 days in 2011, but greatly increased to 348.8 days in 2012. It was rather low, compared to the industrial average ratio, but the period for defend medical costs, so-called liquidity, is vulnerable. In addition, the debt ratio of this hospital kept increasing, from $329.1 \%$ and $510.1 \%$ to $872.1 \%$ for the three years. Since the debt ratio exceeded $200 \%$, such an excessive debt ratio functions as a loss factor by greatly increasing financial costs. Particularly, the debt ratio is higher than the industrial average ratio, this hospital's capability of redeeming long-term debts becomes weakened more and more. At the same time, the equity capital ratio kept decreasing, from 23.3\% in 2010 and $16.4 \%$ in 2011 to $10.3 \%$ in 2012. Since it is lower than the industrial average ratio, this hospital is judged to have a poor financial stability. The interest earned ratio kept sharply increasing, from 10.7 times in 2010 to 48 times in 2011, but it greatly decreased to 8.7 times in 2012, so it was analyzed that this hospital doesn't have an ability good enough to pay for interest costs. The fixed ratio of this hospital exceeded $100 \%$ for the three years, which indicates that this hospital engage in debt in addition to its equity 
capital for fixed asset investments. As the fixed assets to long-term capital ratio is below 100\%, investments seemed to be made into fixed assets within the range of equity capital and fixed liabilities, and as it is lower than the industrial average ratio, this study evaluated that this hospital has properly sustained its stability of capital allocation.

Since DOL is -10 , the medical return will decrease by $10 \%$ when the medical profits decrease by $1 \%$. The higher the medical return gets, the lower the operating leverage becomes. In other words, as the medical profits increase, the effect of fixed medical costs gradually decreases, leading to a reduction in the operating leverage effect. However, the fixed assets of this hospital have a low level of generating the fixed medical costs. Since DFL is 0.9 , the net profit increases by $0.9 \%$ when the medical return increases by $1 \%$. As the financial leverage is smaller than 1 , it is clear that there is no financial leverage effect, which expands the fluctuation range of net profits rather than that of medical profits. Lastly, as DOL and DFL are -10 and 0.9 respectively, DCL is as low as -0.9 , so there hardly appears combined leverage effect.

* (DOL: Degree of operating leverage, DFL: Degree of financial leverage, DCL: Degree of combined leverage)

\section{Conclusion}

Based on 3 years' data from 2010 to 2012, provided by accessible sites of public organizations, such as Alio(www.alio. go.kr), a public organization information, Health Insurance Review \& Assessment Service (www.hira.or.kr), Ministry of Health and Welfare(www.mw.go.kr) and an upper-level general hospital in C Province, this study analyzed the ordinary characters of an upper-level general hospital system, and its common-type balance sheet, common-type profit and loss statement and financial ratio. The results can be summarized as below.

Firstly, out of all the 1,069 hospital staff, there were 272 doctors working for 24 medical departments, out of whom the majority was 33 physicians. Most of the nurses were third-class ones, and about 2,000 outpatients and 600 inpatients on average were treated per day.

Secondly, as a result of analyzing the common-type balance sheet, this study found out that intangible assets out of the fixed assets accounted for $41 \%$, the majority, out of which usable and profitable donation asset buildings were of great importance, and the liquid assets increased more in 2012 than 2011. In the financial structure, the ratio of liquid liabilities was over $50 \%$ out of all the liabilities in 2012, and the ratio of purchase payables was high as well. The ratio of fixed liabilities reached up to $40 \%$, out of which the retirement benefit appropriation fund was quite high. The capital was over $80 \%$, but the surplus was in a deficit state. Compared to the capital, the ratio of total liabilities was about $90 \%$, which indicates the financial structure of this general hospital was vulnerable.

Thirdly, as a result of analyzing the common-type profit and loss statement, this study found out that the medical profits from inpatients were higher than profits from outpatients. The material cost was related to the medical quality of this 
general hospital, and it was as high as $30 \%$ out of the total costs and was about $45 \%$ of the labor cost. This general hospital showed $10 \%$ in the ratio of non-medical profits, and it seemed because of government subsidies. The ratios of medical profits and current net income were gradually changing for the better in 2012, compared to 2011.

Lastly, as a result of analyzing the financial ratio, this study discovered that the liquidity ratio kept decreasing, from $110.7 \%$ in 2010 and $102.0 \%$ in 2011 to $77.2 \%$ in 2012. Besides, it was analyzed that the liquidity ratio and the net working capital ratio greatly decreased, while the quick ratio and the liquid ratio kept decreasing.

\section{References}

1. Yang DH, Kam HK, Jung DC. Hospital business analysis, 2011;08:22-23.

2. Jung KS. Hyundai of Hospital Business Analysis, 2005;08:4-7.

3. (www.alio.go.kr).

4. (www.hira.or.kr).

5. (www.mw.go.kr).

6. http://www.khidi.or.kr.

7. http://cafe.daum.net/terminology.

8 Gapenski LC, Vogel WB. The Determinants of Hospital Profitability. Hospital \& Health Administration, 1993;38(1): 34-45.

9. Lee EH, Jung KS, et al. A Case Study of Hospital Business Analysis. Journal of Korea Sociely of hospital Administration, 2012;17(1):79-112.

10. Yang JH. An Analysis of the Relationship between Publicness and Profitability of National University Hospitals.
Journal of Korea Society of hospital Administration, 2013;18(3):43-61.

11. Ahn IW, Yang DH. An Investigation of Factors Affecting Management Efficiency in Korean General Hospitals Using DEA Model. Korean Journal of Hospital Management, 2005;01:71-92.

12. www.hanaif.re.kr. Business Environment Evaluation and Regional Profitability Analysis of Domestic Hospital Industry 2010.06.11.

13. Kim JK, Jeon JW. Static and Dynamic Analysis of Efficiency of Korean Regional Public Hospitals. Journal of Korea Society of hospital Administration, 2010; 15(1):27-48.

14. Jeong SW, Inoue YU, Seo YJ, Kim YH. Managerial Effectiveness of Integrated Delivery System in Japan. Journal of Korea Society of hospital Administration, 2009; 14(2):60-74.

15. Shin DW, Shin CG, Jung KT. A Study on Quality-incorporating Models in Evaluation of Hospital Efficiency with Data Envelopment Analysis. Journal of Korea Society of hospital Administration, 2008; 13(3):69-93.

16. Park BT, Lee DH. An Analysis of Efficiency in Major University Hospitals in Domestically Capital Area Through DEA Analysis. Journal of Korea Society of hospital Administration, 2011; 16(4): 35-66. 